\title{
THE COPLANAR WAVEGUIDES WITH FINITE METAL THICKNESS AND CONDUCTIVITY
}

\author{
Jeng-Yi Ke and Chun Hsiung Chen \\ Department of Electrical Engineering, \\ National Taiwan University, \\ Taipei, Taiwan 10617, ROC
}

\begin{abstract}
A new method of modifying the conventional spectral-domain approach (SDA) is proposed to deal with the coplanar waveguide in which the thickness and conductivity of signal strip and ground planes are finite. With this method, the unknowns are constrained in the slot regions and the CPU time may be much reduced. In this study, the effective dielectric constant and attenuation constant are carefully studied.
\end{abstract}

\section{INTRODUCTION}

Previous analysis of microwave planar guiding structures is usually conducted under the assumptions of infinitely thin metal strip and infinite conductivity. In millimeter-wave regime, however, the problem of finite metal thickness and finite conductivity must be taken into consideration, because the metal thickness may be comparable to the skin depth of the signal strip and the ground planes. Especially for coplanar waveguides, the effect of lossy conductor on both effective dielectric constant and attenuation constant is not minor [1].

The characteristics of coplanar waveguides with finite metal thickness and conductivity were discussed by the conventional perturbation techniques incorporated with the extended spectraldomain approach [2]-[3] and the transverse resonance technique [4]. They were also discussed by the full-wave techniques, such as the mode matching [1],[5] and the method of lines [6]. Howev- er, the perturbation approach will not be suitable whenever the skin depth and the metal thickness are of the same order, and the above-mentioned full-wave techniques are tirne consuming. In this study, a new method is proposed to deal with the coplanar waveguide (Fig.1(a)) in which the thickness and conductivity of signal strip and ground planes are finite. Here, the spectral domain approach (SDA) is modified to adapt the case with finite metal thickness and conductivity. By this new method, the integration along $y$-direction is analytically integrated. Hence it is as easy as the conventional SDA in which only one-dimensional integration has to be performed to get the matrix equation for the propagation constant.

\section{FORMULATION}

The cross section of coplanar waveguide is shown in Fig.1(a). To solve this problem, the equivalent structure shown in Fig.1(b) is investigated, in which the slots are filled with the conductor of conductivity $\sigma$ and an equivalent current $\mathbf{J}=\left(y_{0}-y_{b}\right) \mathbf{E}$ is introduced in the slot regions, where $y_{0}=j \omega \epsilon_{0}$ and $y_{b}=j \omega \epsilon_{0}+\sigma$. The relation between the electric field $\mathbf{E}(\mathbf{r})$ and the equivalent current density $\mathbf{J}(\mathbf{r})$ within the slot regions $\Omega$ is

$$
\begin{aligned}
\mathbf{E}(\mathbf{r}) & =\int_{\Omega} \overline{\overline{\mathbf{G}}}\left(\mathbf{r}-\mathbf{r}^{\prime}\right) \bullet \mathbf{J}\left(\mathbf{r}^{\prime}\right) \mathbf{d} \mathbf{r}^{\prime} \\
& =\left(y_{0}-y_{b}\right) \int_{\Omega} \overline{\overline{\mathbf{G}}}\left(\mathbf{r}-\mathbf{r}^{\prime}\right) \bullet \mathbf{E}\left(\mathbf{r}^{\prime}\right) \mathbf{d} \mathbf{r}^{\prime}
\end{aligned}
$$

Here, $\overline{\bar{G}}$ is the dyadic Green's function for the layer structure of Fig. 1(c). It should be emphasized that the conductor is now regarded as a lossy layer therefore the effect of lossy signal strip and ground planes may be discussed through 


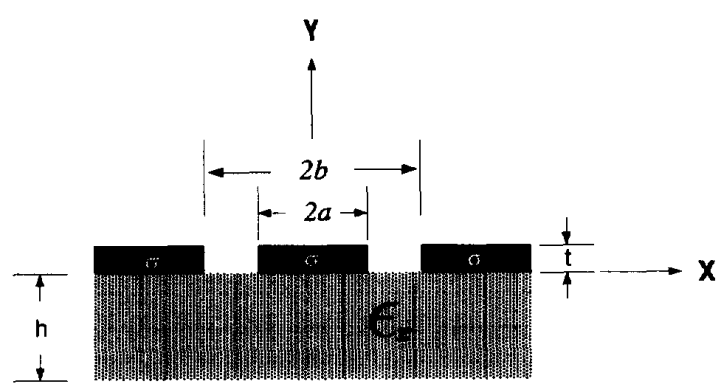

(a)

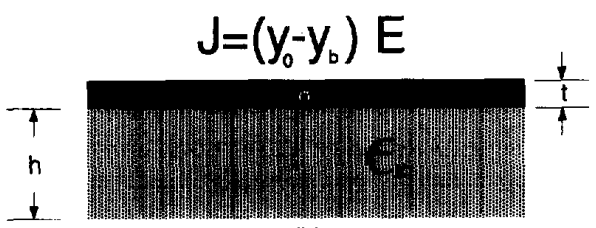

(b)

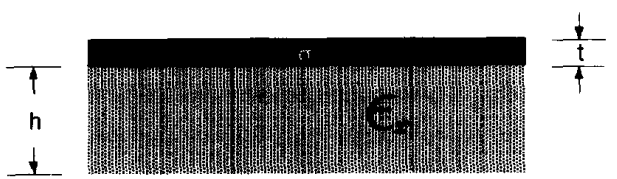

(c)

Fig. 1: Cross section of coplanar waveguide.

these Green's functions. By weighting both sides of (1) by any arbitrary function $\mathbf{w}(\mathbf{r})$ and then integrated, one may get a two-dimensional integral equation. Because the $y$-dependence form of the spectral-domain Green functions is a linear combination of $\exp (j \beta y)$ and $\exp \left(j \beta y^{\prime}\right)$, thus, if the bases of $\mathbf{E}$ are properly chosen, the integral equation can be reduced to the one-dimensional one as in the conventional SDA. In this study, the fields within the slot regions are represented by

$$
\begin{aligned}
& E_{z}(x, y)=\sum_{i=0}^{m} \sum_{j=0}^{n} a_{i j} \psi_{z}^{i}(x) \phi_{z}^{j}(y) \\
& E_{x}(x, y)=\sum_{i=0}^{m} \sum_{j=0}^{n} b_{i j} \psi_{x}^{i}(x) \phi_{x}^{j}(y) \\
& E_{y}(x, y)=\sum_{i=0}^{m} \sum_{j=0}^{n} c_{i j} \psi_{y}^{i}(x) \phi_{y}^{j}(y),
\end{aligned}
$$

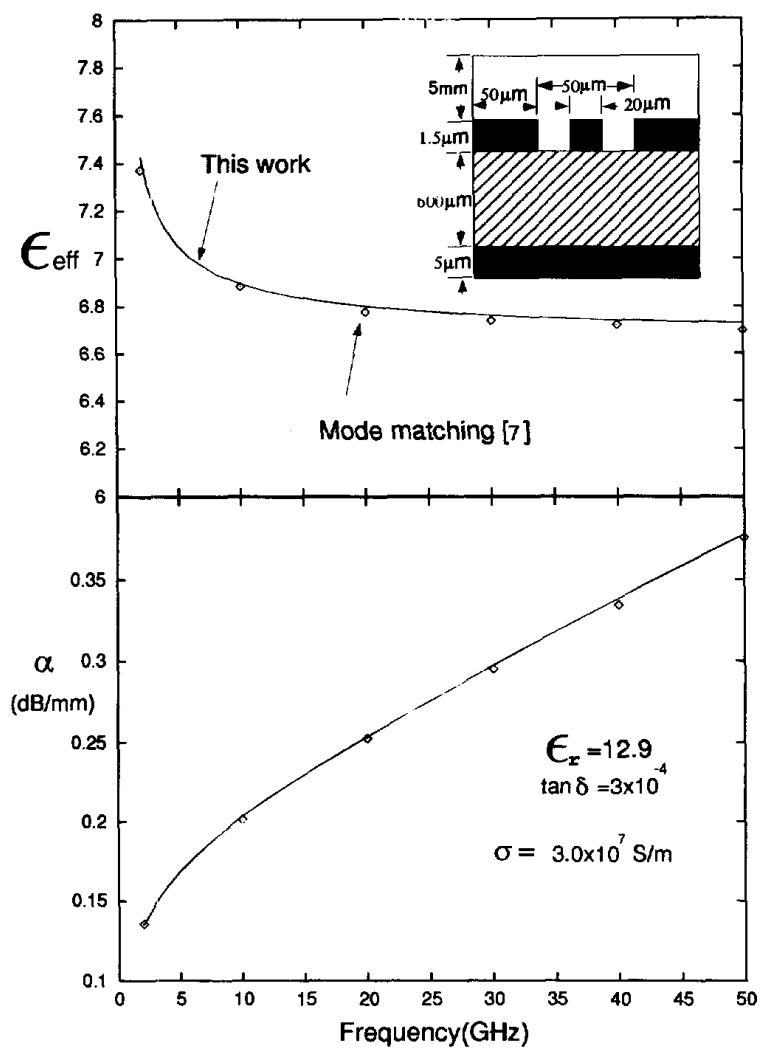

Fig. 2: Comparison of effective dielectric constant $\epsilon_{\text {eff }}$ and attenuation constant $\alpha$ with those of mode matching.

where $\psi_{p}^{l}(x)$ is the Legendre polynomial and $\phi_{p}^{l}(y)$ is the piecewise linear function.

\section{NUMERICAL RESULTS}

Numerical results such as effective dielectric constant $\epsilon_{e f f}=\beta^{2} / k_{0}^{2}\left(k_{0}^{2}=\omega^{2} \mu_{0} \epsilon_{0}\right)$ and attenuation constant $\alpha$ are carefully studied. To check the accuracy of the new approach's results, the frequency-dependent $\epsilon_{e f f}$ and $\alpha$ for a shielded structure are shown in Fig. 2 with those of [7] also included for comparison. Agreement in both results is observed.

Fig. 3 shows the effect of increasing metal thickness $t$ on the effective dielectric constant and attenuation constant. As expected, both 


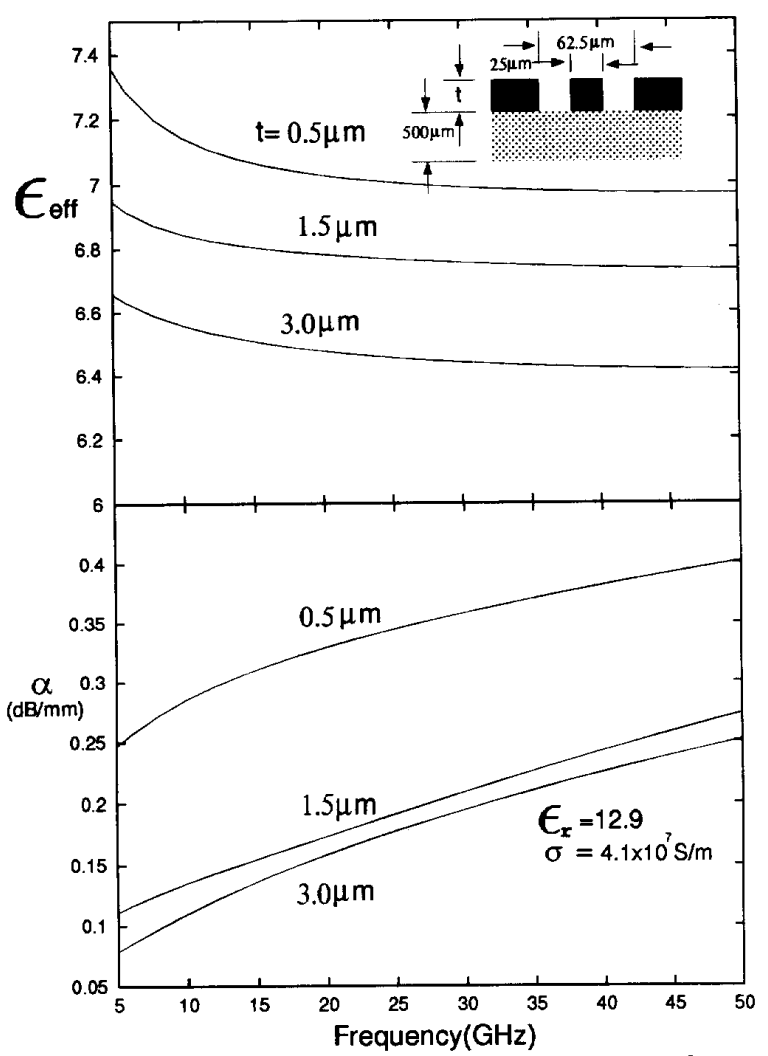

Fig. 3: Effective dielectric constant $\epsilon_{\text {eff }}$ and attenuation constant $\alpha$ versus frequency with metal thickness $t$ as parameters.

the effective dielectric constant and attenuation constant decrease as $t$ increases. It should be notes that the slope of $\epsilon_{\text {eff }}$ in low frequency range is negative. When the thickness-to-skin depth ratio $t / \delta$ approaches infinity, the current is flowed essentially along the metal surface and the dispersion curve grows as the frequency increases as predicted by the conventional technique of assuming infinite conductivity. However when $t$ and $\delta$ are of the same order, the current can not be assumed as a surface current, and this will introduce an internal inductance which then increases the effective dielectric constant in low frequency [1].

Shown in Fig. 4 is the effect of increasing aperture width $b$. Also included in this figure are the experimental results of [8]. Good agreement among these results is observed. Noteworthy is the fact that as the aperture width increases, the effective dielectric constant and the attenuation

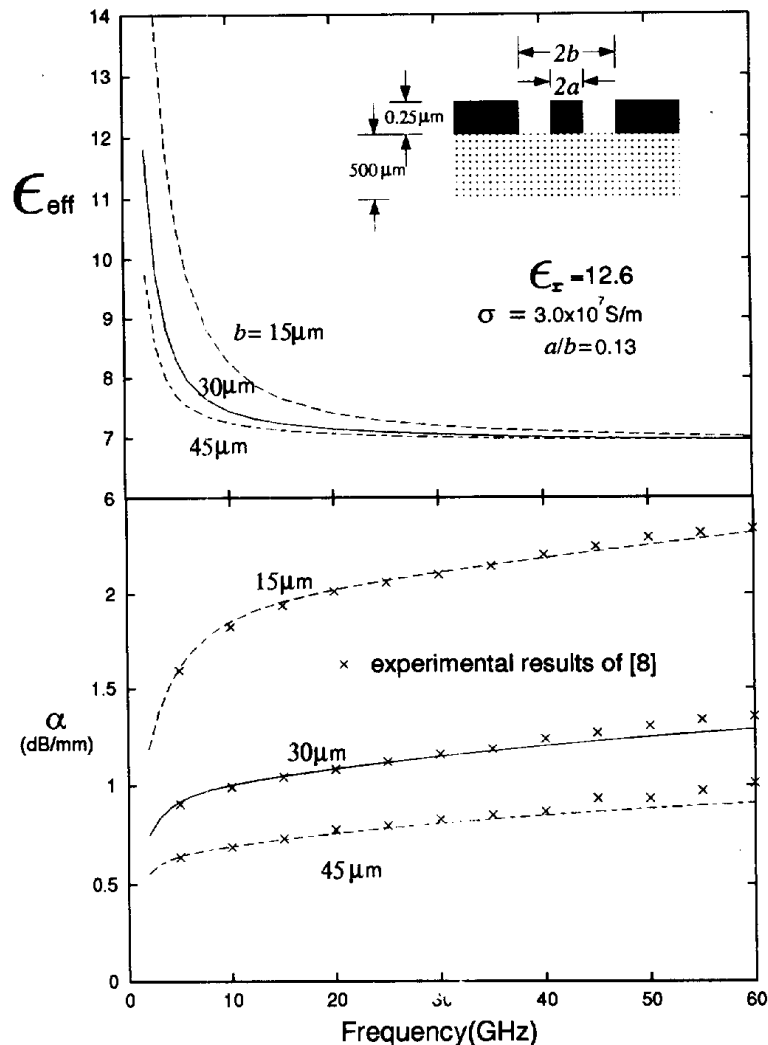

Fig. 4: Effective dielectric constant $\epsilon_{\text {eff }}$ and attenuation constant $\alpha$ versus frequency with aperture width $b$ as parameters.

constant decrease. Especially, as frequency grows, the difference in the effective dielectric constants is decreased. However, the variation in attenuation from changing $b$ is rather obvious.

The effect of varying $a / b$ is shown in Fig. 5. It is interesting to point out that the loss for $a / b=0.4$ is smallest when the frequency is larger than $15 \mathrm{GHz}$. To reduce the conductor loss, the signal strip and the slot width should be of the same order.

\section{CONCLUSIONS}

The advantage of this method is that the only unknowns are constrained in the slot regions and the CPU time may be much reduced in comparison with the other full-wave methods. Besides, the structure may be open or close, the conduc- 


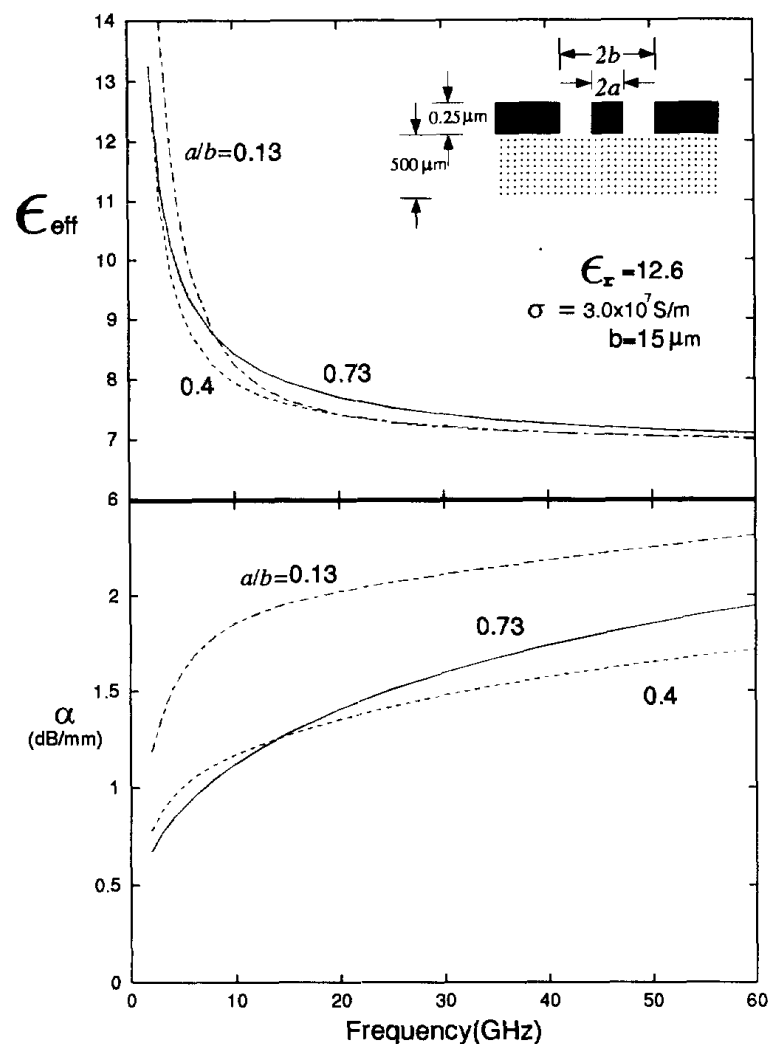

Fig. 5: Effective dielectric constant $\epsilon_{\text {eff }}$ and attenuation constant $\alpha$ versus frequency with $a / b$ as parameters.

tivities of signal strip and ground planes may be different, and the conductor may be replaced by superconductor, semiconductor, or dielectric. In this work, the effective dielectric constant and attenuation constant are discussed in detail.

\section{Acknowledgment}

This work was supported by the National Science Council of the Republic of China under Grant NSC 83-0404-E-002-044.

\section{References}

[1] W. Heinrich, "Full-wave analysis of conductor losses on MMIC transmission lines," IEEE Trans. Microwave Theory Tech., vol. 38 , no. 10 , pp. $1468-1472$, Oct. 1990.
[2] T. Kitazawa and T. Itoh, "Propagation characteristics of coplanar-type transmission lines with lossy media," IEEE Trans. Microwave Theory Tech., vol. 39, no. 10, pp. 1694-1700, Oct. 1991.

[3] T. Kitazawa, D. Polifko, and H. Ogawa, "Analysis of CPW for $\mathrm{LiNbO}_{3}$ optical modulator by extended spectral domain approach," IEEE Microwave Guided Wave Lett., vol. 2, no. 8, pp. 313-315, Aug. 1992.

[4] F. Alessandri, G. Baini, G. D'Inzeo, and R. Sorrentino, "Conductor loss computation in multiconductor MIC's by transverse resonance technique and modified perturbational method" IEEE Microwave Guided Wave Lett., vol. 2, no. 6, pp. 250-252, June 1992.

[5] C. -C. Tien, C. C. Tzuang, and S. T. Peng, "Effect of finite-width backside plane on overmoded conductor-backed coplanar waveguide," IEEE Microwave Guided Wave Lett., vol. 3, no. 8, pp. 259-261, Aug. 1993.

[6] K. Wu, R. Vahldieck, J. L. Fikart, and $\mathrm{H}$. Minkus, "The influence of finite conductor thickness and conductivity on fundamental and higher-order modes in miniature hybrid MIC's (MHMIC's) and MMIC's," IEEE Trans. Microwave Theory Tech., vol. 41, no. 3, pp. 421-430, March 1993.

[7] W. Heinrich, "Conductor loss on transmission lines in monolithic microwave and millimeter-wave integrated circuits," International Journal of Microwave and Millimeter-Wave Computer-Aided Engineering, vol. 2, no. 3, pp. 15j-167, 1992.

[8] W. H. Haydl, "Experimentally observed frequency variation of the attenuation of millimeter-wave coplanar transmission lines with thin metallization," IEEE Microwave Guided Wave Lett., vol. 2, no. 8, pp. 322324, Aug. 1992. 